R. Kreienberg ${ }^{\mathrm{a}}$ M. Freund ${ }^{\text {b }}$

${ }^{a}$ Universitäts-Frauenklinik und Poliklinik, Ulm

${ }^{\mathrm{b}}$ Abteilung Hämatologie/Onkologie, Klinik und Poliklinik für Innere Medizin, Universität Rostock

\section{Clinical Outcome of Topotecan: New Strategies Play an Important Role in the Treatment of Lung Cancer, Other Solid Tumors, and Hemoblastosis}

The symposium focused on the current use of and new indications for the semisynthetic camptothecin analogue topotecan $\left(\right.$ Hycamtin $\left.{ }^{\circledR}\right)$. Camptothecins represent a novel class of antineoplastic agents directed at a new target, topoisomerase I. After the identification of the target by Liu and colleagues, the rapid development of camptothecin and its analogues began. Clinical activity of topotecan has been proven for a range of tumors, especially for ovarian and lung cancer. The incorporation of taxanes into first-line therapy of advanced ovarian cancer led to the question of the optimum treatment in recurrent disease. Final results of the phase III comparison between paclitaxel and topotecan therapy after failure of platinum treatment in advanced ovarian cancer show no statistically significant difference in survival, progression-free intervals and remission rates for both agents. Further studies evaluated topotecan therapy in patients previously treated with paclitaxel and a platinum compound. Responses were seen even in the refractory situation and the presented data on long-term application of topotecan underline the subjectively well-tolerated therapy with substantial clinical benefit for the patients. Additionally, combination therapies with topotecan show promising results not only in recurrent situations but also in first-line treatment as demonstrated by the spectrum of phase I and II data presented at the last ASCO meeting.

In small-cell lung cancer topotecan has undergone the most extensive evaluation of all new chemotherapy agents. It is clearly active in second-line treatment, and shows promising results in first-line therapy. Additional benefit lies in the pene- tration of the intact blood brain barrier, because thus topotecan has access to a 'sanctuary' generally not accessible to cytostatics. It may be the drug of choice in induction regimes due to the fact that it may prevent the high rate of up to $80 \%$ of brain metastases seen in these patients under effective chemotherapy.

Topotecan is in an earlier stage of development in non-smallcell lung cancer. Only a few studies with a small number of patients have been completed and published.

The preclinical and clinical development of the topoisomerase I inhibitor topotecan is also ongoing in sarcoma. Evaluation in phase I and II studies showed antitumor activity in neuroblastoma and in metastatic rhabdomyosarcoma.

The enzyme topoisomerase I was detected in the nucleolus of all cells, but its concentration is greatly increased in many neoplastic tissues, including colon adenocarcinoma, nonHodgkin's lymphoma, and acute myeloid and lymphocytic leukemias.

In myelodysplastic syndromes, chronic myelomonocytic leukemia and multiple myeloma, topotecan demonstrated good activity with acceptable toxicity. Thus far, the present treatment results with topotecan in hematological malignancies give cause to optimism; however, the full extent for topotecan use is yet to be determined.

In summary, the symposium intended to show that there is a new compound with favorable clinical outcome and, based on this data, it gives the prospects for the ultimate implementation of these clinical advances into practice.

\begin{tabular}{ll}
\hline KARGER & ๑1998 S. Karger GmbH, Freiburg \\
& Fax (0761) 4520714 \\
www.karger.com \\
Accessible online at: \\
http://BioMedNet.com/karger
\end{tabular}

